\section{O efeito da amamentação na massa óssea de mulheres na pós-menopausa: revisão sistemática de estudos observacionais}

\section{The effect of breastfeeding on the bone mass of post-menopausal women: a systematic review of observational studies}

Adriany Cristine Santos Gonçalves 1 Marina de Figueiredo Ferreira 2 Maria Helena Hasselmann 3 Eduardo Faerstein 4

1,2 Pós-graduação em Nutrição. Instituto de Nutrição. Universidade do Estado do Rio de Janeiro. Rua São Francisco Xavier, $n^{\circ} 524$. Bloco D, $12^{\circ}$ andar, sala 12024. Maracanã. Rio de Janeiro, RJ, Brasil. CEP: 20.559-900. E-mail: drik_cristine@yahoo.com.br

3 Departamento de Nutrição Social. Instituto de Nutrição. Universidade do Estado do Rio de Janeiro. Rio de Janeiro, RJ, Brasil. 4 Departamento de Epidemiologia. Instituto de Medicina Social. Universidade do Estado do Rio de Janeiro. Rio de Janeiro, RJ, Brasil.

\begin{abstract}
Objectives: to evaluate the effect o breastfeeding on the bone mass of post-menopausal women.

Methods: a systematic review of observational studies was carried out. The literature was searched using the Lilacs, Medline and Scopus bibliographical databases, in June 2015, with no restrictions as to year of publication. To be included in the review, the article must have investigated the history of breastfeeding and post-menopausal bone mass and references were checked to unearth further studies. Two reviewers independently extracted data and evaluated the potential bias of the studies.

Results: 34 studies were selected, six of which (26.5\%) found that breastfeeding significantly increased bone mass and 15 (44\%) came up with the opposite finding. There was much variation among the studies as to study population, measurement of exposure and location of bone mass measurement, making it difficult to compare the results.

Conclusions: the marked heterogeneity of the studies made it difficult to conclude the direction of association between breastfeeding and bone mass in post-menopausal women. More methodologically rigorous studies are needed to assess this issue of great importance for clinical practice and public health.

Key words Breastfeeding, Bone, Bone density, Postmenopause
\end{abstract}

\section{Resumo}

Objetivos: avaliar o efeito da amamentação na massa óssea de mulheres na pós-menopausa.

Métodos: trata-se de revisão sistemática de estudos observacionais. A pesquisa da literatura foi realizada nas bases de dados bibliográficos Lilacs, Medline e Scopus, em junho de 2015, sem restrição quanto ao ano de publicação. Para ser incluído nesta revisão, o artigo deveria avaliar o histórico de amamentação e a massa óssea na pós-menopausa, sendo suas referências checadas para busca de novos estudos. Dois revisores, de forma independente, realizaram a extração de dados e a avaliação da suscetibilidade a vieses dos estudos.

Resultados: foram selecionados 34 estudos, dos quais seis (26,5\%) mostraram que a prática de amamentação aumentou significativamente a massa óssea e 15 (44\%) encontraram o inverso. Constatouse grande diversidade entre os estudos quanto à população de estudo, mensuração da exposição e sítios de avaliação da massa óssea, dificultando a comparabilidade entre os resultados.

Conclusões: a marcante heterogeneidade dos estudos torna inconclusiva a direção da associação entre a amamentação e a massa óssea em mulheres na pós-menopausa, sendo necessários estudos com maior rigor metodológico para avaliar esse problema de grande relevância para as áreas clínica e de saúde pública.

Palavras-chave Amamentação, Osso, Densidade óssea, Pós-menopausa 


\section{Introdução}

A osteoporose é considerada um problema de saúde pública mundial, cuja ocorrência vem aumentando em função do maior número de idosos na população.1-5 Trata-se de uma doença osteometabólica com fragilidade progressiva e debilitante do esqueleto, por redução da massa óssea. ${ }^{2}$ Estima-se que $40 \%$ das mulheres na pós-menopausa terão ao menos uma fratura por osteoporose. ${ }^{6-10}$ Além da idade e de características de vida reprodutiva,11-19 existem dois momentos fisiológicos durante os quais alterações hormonais influenciam o metabolismo ósseo e podem gerar aumento da perda de massa óssea em mulheres: amamentação e menopausa. 20

Durante a amamentação, ocorre intensa desmineralização da massa óssea materna, devido ao requerimento de cálcio aumentado para a produção de leite.21-23 Em condições normais, espera-se que a massa óssea perdida seja restabelecida entre 12 a 18 meses após o parto, quando também ocorrem o desmame e cessa a amenorréia.22,24 $\mathrm{Na}$ menopausa, a perda óssea é intensificada novamente; na fase de transição, essa perda pode chegar a $10 \%$ ao ano, enquanto nos homens é mais gradual $(0,5 \%$ ao ano) e tardia (aproximadamente aos 70 anos). 25

Há divergências entre os estudos que investigaram se a prática da amamentação influencia a massa óssea na pós-menopausa: alguns observaram existir associação direta,16,18,26-28 outros, inversa 12,29-32 e ainda, alguns relataram ausência de associação.13,33-35 Uma revisão da literatura confirmou a inconsistência de resultados. 36

Tendo em vista a alta relevância clínica e de saúde pública do tema, considerou-se relevante investigar em maior profundidade aspectos conceituais e metodológicos dos estudos sobre o tema, atualizando o período de revisão da literatura e enfatizando a avaliação da qualidade dos estudos. Ao investigar, em revisão sistemática, o efeito da amamentação na massa óssea de mulheres na pósmenopausa, pretende-se contribuir para o planejamento de futuras pesquisas e para construção de estratégias de promoção e proteção da saúde óssea da mulher durante a amamentação.

\section{Métodos}

Trata-se de estudo do tipo revisão sistemática da literatura, baseado nos procedimentos produzidos pelo PRISMA. 37 A pesquisa foi realizada com consulta às seguintes bases eletrônicas: Lilacs, Medline via Pubmed e Scopus.
Além da pesquisa eletrônica, realizou-se busca de artigos nas referências dos estudos selecionados. A consulta às bases ocorreu em junho de 2015, sem restrição para o ano de publicação. Os termos $(\mathrm{MeSH}$ Terms) e palavras-chave empregados de forma combinada foram (breastfeeding OR breast feeding $O R$ exclusive breastfeeding) AND (bone $O R$ osteoporosis OR osteoporosis post-menopausal OR metabolic bone diseases) AND (post-menopause OR postmenopausal period).

Estudos observacionais, que avaliaram o histórico da prática da amamentação e sua relação com a massa óssea na pós-menopausa, foram incluídos nesta revisão. Investigações sobre fratura óssea e condição óssea de ex-atletas foram excluídas, e foram considerados apenas artigos em inglês, espanhol e português.

A identificação e a seleção dos artigos foram realizadas por dois pesquisadores através de avaliação dos títulos e resumos, de forma independente. Sempre que os dois revisores concordavam que o título e o resumo não se adequavam aos critérios de elegibilidade, os artigos eram excluídos. Quando existia dúvida, o artigo completo era lido em separado pelos dois revisores e, após nova reunião, era decidido se este atendia aos critérios de inclusão.

Os dados dos estudos - primeiro autor, ano do estudo, local do estudo, tamanho da amostra, idade, mensuração da amamentação e tempo médio de duração da amamentação, o método e sítio ósseo mensurado e desfecho - foram extraídos por dois pesquisadores, de forma independente, em formulário padronizado criado para esta revisão. As diferenças entre os revisores com relação à extração foram resolvidas por meio de discussão e releitura do artigo.

Para a descrição da susceptibilidade a vieses, foi elaborada pelos autores uma checklist com itens abordando questões relativas aos métodos (critérios de elegibilidade, inclusão de variáveis como: etnia, mensuração da duração total do período de lactação, amamentação exclusiva, idade gestacional, tempo entre as gestações, ocorrência de gestação gemelar, hábito fumo/álcool, anos desde a menopausa, atividade física, uso de terapia de reposição hormonal, consumo de cálcio/suplementação, doenças que afetam o metabolismo ósseo e medicamentos que afetam a saúde óssea, e métodos estatísticos), aos resultados (população de estudo, dados descritivos e resultados apresentados) e à discussão (limitações e generalização), com base nas recomendações do Strengthening the Reporting of Observational Studies in Epidemiology (STROBE).38 
O preenchimento dos itens foi expresso como: "sim" ou "não", indicando que a informação foi ou não considerada, respectivamente; ou ainda, "incompleto" (I), quando a informação não estava suficientemente clara.

\section{Resultados}

Foi identificado um total de 195 artigos nas bases eletrônicas consultadas e 11 artigos pela checagem manual das referências dos selecionados. Removidos os artigos repetidos, realizou-se a leitura dos títulos e resumos de 160 trabalhos. Destes, 42 foram triados para leitura na íntegra. Posteriormente, oito artigos foram excluídos por não corresponderem aos critérios de elegibilidade. Por fim, foram incluídos 34 artigos (Figura 1).

Dentre os artigos revisados, 33 apresentam delineamento seccional12,13,16-19,26-35,39-55 e um foi estudo de coorte.56 A origem dos estudos se distribuiu entre: Estados Unidos da América - EUA $(n=8)$, Turquia $(n=7)$, China $(n=3)$, Dinamarca $(\mathrm{n}=2)$, Japão $(\mathrm{n}=2)$, Itália $(\mathrm{n}=1)$, Holanda $(\mathrm{n}=1)$, Suécia $(n=1)$, Grécia $(n=1)$, Sri Lanka $(n=1)$, Irã $(\mathrm{n}=1)$, Reino Unido $(\mathrm{n}=1)$, Caribe $(\mathrm{n}=1)$, México $(\mathrm{n}=1)$, Israel $(\mathrm{n}=1)$ e Coréia $(\mathrm{n}=1)$. Um dos estudos foi multicêntrico e envolveu Dinamarca, Inglaterra e EUA $^{34}$ (Tabela 1).

Figura 1

Fluxograma de seleção dos artigos para a revisão sistemática

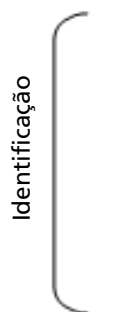

Artigos identificados através de pesquisa nas bases de dados $(n=195)$

늄

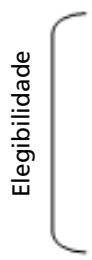



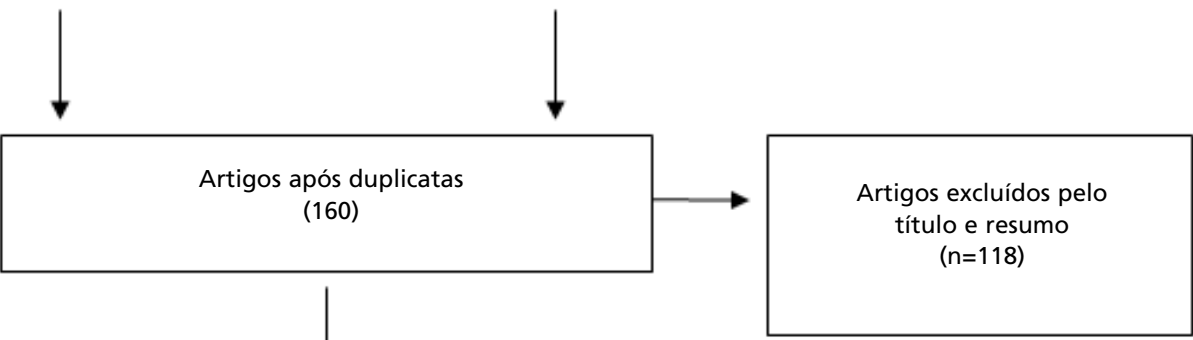

Artigos excluídos: - não apresentaram os resultados para o grupo de interesse $(n=5)$

- métodos diferentes de mensuração da massa óssea $(n=1)$;

- não avaliou a massa óssea $(n=2)$ 
Tabela 1

Características dos estudos observacionais que avaliaram o efeito da amamentação na massa óssea de mulheres na pós-menopausa.

\begin{tabular}{|c|c|c|c|c|c|}
\hline $\begin{array}{l}\text { Referência, ano } \\
\text { (Local do estudo) }\end{array}$ & $\mathbf{N}$ & $\begin{array}{l}\text { Idade (anos) } \\
\text { faixa etária e/ou } \\
\text { média (dp) }\end{array}$ & $\begin{array}{l}\text { História de lactação } \\
\text { (Tempo médio) }\end{array}$ & $\begin{array}{l}\text { Método (sítio de } \\
\text { mensuração) } \\
\text { Avaliação de precisão }\end{array}$ & Principais resultados \\
\hline \multicolumn{6}{|l|}{ Seccional } \\
\hline $\begin{array}{l}\text { Aloia et al.391983 } \\
\text { (EUA) }\end{array}$ & 80 & $\begin{array}{l}45-55 \\
52,2 \pm 0,4\end{array}$ & $\begin{array}{l}\text { Sim/Não } \\
\text { (NA) }\end{array}$ & $\begin{array}{l}\text { SPA } \\
\text { (CMO no rádio distal) }\end{array}$ & $\begin{array}{l}\text { A amamentação apresentou } \\
\text { correlação positiva significativa } \\
\text { com o CMO. }\end{array}$ \\
\hline
\end{tabular}

1988 (EUA)

amamentadas por

pelo menos duas

semanas

(NA)

Stevenson et al.41 $1989172 \quad 52,9 \pm 4,6$

(Reino Unido)*

Sim/Não

(NA)

\section{Lissner et al.31 1991}

63

$59,0 \pm 0,97$

(Suécia)

Kritz-Silverstein et
al.42 1992 (EUA)

741

60-89

$\pm 75,4$

Bauer et al.43 1993

9704

(EUA)

Berning et

94

$45,7-60,0$

mediana $=$

52,9

Melton et al.44 $1993 \quad 216 \quad$ NA

(EUA)*
Total em semanas

Total em meses

Sim/Não

(NA)

Sim/Não

(NA)

Sim/Não;

Total em meses e se

amamentou mais de

8 meses ou não

(8 meses)
(CL (L2-L4) e CF entre cabeça e trocanter)

Coeficiente de variação

DPA

(CL - L2-L4, CF, trocanter e região de Ward)

Coeficiente de variação

DPA

(CMO - CL (L3))

DPA(CL (L1 - L4) e quadril

(trocanter, intertrocanter

e cervical)

SPA(Rádio e pulso

ultradistal - braço não

dominante)

SPA

(rádio distal e médio e o

calcâneo)

Coeficiente de variação

TC

(CL (L1-L3), osso trabecular e cortical)

Coeficiente de variação

DPA

( $C L$, trocanter, fêmur) e

CMO (rádio)

Coeficiente de variação significativa entre a lactação e a DMO somente na $\mathrm{CL}$ quando considerado o $n^{\circ}$ partos.

A DMO da CL de mulheres que amamentaram foi maior em relação as que não amamentaram.

Observou que o CMO reduziu com o aumento do tempo de lactação (sem teste estatístico). Sugere o tempo de lactação como preditor do CMO.

A amamentação não mostrou associação com a DMO.

A amamentação não mostrou associação com a DMO.

Existe associação significativa entre a DMO do osso trabecular e o tempo de lactação. E mulheres com tempo de amamentação >24 meses tiveram maior DMO trabecular em relação as que amamentaram $\leq 24$ meses.

Amamentar acima de 8 meses se relacionou à maior $\mathrm{DMO}$ significativamente na $C L$ e fêmur. 
Características dos estudos observacionais que avaliaram o efeito da amamentação na massa óssea de mulheres na pós-menopausa.

\begin{tabular}{|c|c|c|c|c|c|}
\hline $\begin{array}{l}\text { Referência, ano } \\
\text { (Local do estudo) }\end{array}$ & $\mathbf{N}$ & $\begin{array}{l}\text { Idade (anos) } \\
\text { faixa etária e/ou } \\
\text { média (dp) }\end{array}$ & $\begin{array}{l}\text { História de lactação } \\
\text { (Tempo médio) }\end{array}$ & $\begin{array}{l}\text { Método (sítio de } \\
\text { mensuração) } \\
\text { Avaliação de precisão }\end{array}$ & Principais resultados \\
\hline \multirow[t]{7}{*}{ Fox et al.45 1993 (EUA) } & 2230 & $\geq 65(71)$ & Tempo total (meses) e & SPA & diferença \\
\hline & & & $\mathrm{n}^{\circ}$ de crianças & (radio distal e proximal) & significativa na DMO de \\
\hline & & & amamentadas: & & mulheres que amamentaram, \\
\hline & & & (7,4 meses) & & considerando o tempo, em \\
\hline & & & & & relação às que \\
\hline & & & & & amamentaram. A DMO das \\
\hline & & & & & $\begin{array}{l}\text { mulheres que amamentaram } \\
\text { foi levemente maior. }\end{array}$ \\
\hline
\end{tabular}

$\begin{array}{llll}\begin{array}{l}\text { Hu et al.27 } 1994 \\ \begin{array}{l}\text { (China)* } \\ \text { (E) }\end{array}\end{array} & \begin{array}{l}35-75 \\ 59,7 \pm 7,8\end{array} & \begin{array}{l}\text { Total e por criança, } \\ \text { em meses: } \\ \text { (Total }=129,2 \pm 72,1) \\ \end{array} \\ & & \begin{array}{l}\text { (Por criança }= \\ 19,8 \pm 11,4)\end{array} \\ & & \\ \begin{array}{l}\text { Orwoll et al.33 } 1996 \\ \text { (EUA) }\end{array} & 7963 & \geq 65 & \text { Sim/Não } \\ & & 73,8 \pm 5,3 & \text { (NA) }\end{array}$

SPA

(Rádio - braço nãodominante)

Coeficiente de variação

DXA

(CL (L1- L4), quadril total, $\mathrm{CF}$, trocanter, região de Ward, região intertrocantérica)

Coeficiente de variação

Sinigaglia et al.46 1996540 (Itália)

Osei-Hyiaman et al.47 1035
$>12 \mathrm{~m}$ :

$57,3 \pm 4,92$ a

$4 \mathrm{~m}$ :

$56,7 \pm 4,6$ Não

Amamentou:

$56,7 \pm 4,7$

$60,47 \pm 6,14$
SPA

(metacarpo) 1998 (Japão)

Se amamentou ou utilizou fórmula ou ambos

Grupo com

osteoporose

$(48,3 \pm 3,02)$

Sem osteoporose

$(26,6 \pm 1,2)$

Grainge et al.34 $2001 \quad 580 \quad 45-61$

(Dinamarca,

Inglaterra e EUA)

Kojima et al.48 2002

713

(Japão)

\section{Total em semanas \\ (Mediana $=16$ \\ semanas)}

Total em meses

(55-59 anos: $78,1 \pm 50,2$

60-64 anos: $116,0 \pm 69,0$

65-69 anos: $118,0 \pm 93,0$ )
O tempo de amamentação por criança apresentou correlação positiva significativa com a DMO no rádio distal.

O fato da mulher amamentar não está associado a DMO na pós-menopausa.

\begin{abstract}
Não houve diferença significativa na DMO entre mulheres com diferentes tempos de amamentação nem em relação às que nunca
\end{abstract} amamentaram.

Coeficiente de variação

A amamentação exerceu influência na DMO. E mulheres que utilizaram fórmula e amamentaram apresentaram maiores valores de DMO em relação às que só forneceram leite materno.

DXA

(CL, trocanter (osso trabecular), CF, radio/ulna A duração da amamentação não mostrou associação com a DMO.

(osso cortical), corpo inteiro)

DXA

(CL (L2- L4)

Coeficiente de variação
Sem correlação significativa após ajuste para confundidores. Exceto quanto a faixa etária de 60-64 anos em que houve correlação inversa significativa. 
Características dos estudos observacionais que avaliaram o efeito da amamentação na massa óssea de mulheres na pós-menopausa.

\begin{tabular}{|c|c|c|c|c|c|}
\hline $\begin{array}{l}\text { Referência, ano } \\
\text { (Local do estudo) }\end{array}$ & $\mathbf{N}$ & $\begin{array}{l}\text { Idade (anos) } \\
\text { faixa etária e/ou } \\
\text { média (dp) }\end{array}$ & $\begin{array}{l}\text { História de lactação } \\
\text { (Tempo médio) }\end{array}$ & $\begin{array}{l}\text { Método (sítio de } \\
\text { mensuração) } \\
\text { Avaliação de precisão }\end{array}$ & Principais resultados \\
\hline $\begin{array}{l}\text { Zhang et al.49 } 2003 \\
\text { (China) }\end{array}$ & 214 & $60,35(5,74)$ & $\begin{array}{l}\text { Total em meses: } \\
7,35(5,36) \text { meses }\end{array}$ & $\begin{array}{l}\text { DXA } \\
\text { (CL, quadril total) } \\
\text { Coeficiente de variação }\end{array}$ & $\begin{array}{l}\text { Não houve associação } \\
\text { significativa da DMO com a } \\
\text { duração da amamentação. }\end{array}$ \\
\hline $\begin{array}{l}\text { Gur et al.32 } 2003 \\
\text { (Turquia) }\end{array}$ & 509 & $\begin{array}{l}40-80 \\
61,5 \pm 7,5\end{array}$ & $\begin{array}{l}\text { Total em meses } \\
(103,3 \pm 58,1)\end{array}$ & $\begin{array}{l}\text { DXA } \\
\text { (CL e quadril (colo, } \\
\text { trocanter, região de Ward) } \\
\text { Coeficiente de variação }\end{array}$ & $\begin{array}{l}\text { Sugere que quanto maior o } \\
\text { tempo da amamentação, } \\
\text { menor é a DMO. E mulheres } \\
\text { que não amamentaram tinham } \\
\text { maior DMO em relação às que } \\
\text { amamentaram. }\end{array}$ \\
\hline
\end{tabular}

\begin{tabular}{|c|c|c|c|}
\hline $\begin{array}{l}\text { Hassa et al.35 } 2005 \\
\text { (Turquia) }\end{array}$ & 267 & NA & $\begin{array}{l}\text { Sim/Não } \\
\text { (NA) }\end{array}$ \\
\hline
\end{tabular}

(CL (L1 - L4), fêmur esquerdo (colo,

Não existe associação entre a DMO e a amamentação.

intertrocantérica, região de Ward))

$\begin{array}{lllll}\text { Ozdemir et al.12 } 2005 & 303 & 43-80 & \text { Total em meses } & \text { DXA } \\ \text { (Turquia) } & & 59,5 \pm 8,2 & (13,75 \pm 8,99) & \text { (CL }(\text { L }\end{array}$

DXA

Observou correlação inversa significativa entre o tempo de região de Ward) lactação e a DMO do trocanter, CF e região de Ward.

$\begin{array}{lcll}\begin{array}{l}\text { Dursun et al.17 2006 } \\ \text { (Turquia) }\end{array} & 1486 & >40^{*} & \begin{array}{l}\text { Total em meses } \\ (30,8 \pm 32,8)\end{array} \\ \begin{array}{l}\text { Hill et al.28 } 2008 \\ \text { (Caribe) }\end{array} & 340 & \geq 50 & \begin{array}{l}\text { Sim/Não } \\ \text { (NA) }\end{array} \\ \begin{array}{l}\text { Lenora et al.13 } 2009 \\ \text { (Siri Lanka) }\end{array} & 210 & 45,8-97,7 & \begin{array}{l}\text { Total em meses } \\ (61,3 ; \text { IC } 95 \% 54,5-\end{array} \\ & & 64,6 \pm 8,7 & 68,2)\end{array}$

DXA (CL, CF)

DXA

(quadril esquerdo, subregião do $\mathrm{CF}$ )

DXA

(CL (L2 - L4), CF não dominante)

Coeficiente de variação

$\begin{array}{llll}\text { Hosseinpanah et al.50 } & 245 & 40-80 & \text { Total em meses } \\ 2010 \text { (Irã) } & & 57,5 \pm 6,9 & (68,3 \pm 57,1)\end{array}$

DXA

(CL ( L2-L4), quadril total (trocanter, região de Ward, CF))

\begin{tabular}{|c|c|c|c|c|}
\hline $\begin{array}{l}\text { Schnatz et al.16 } 2010 \\
\text { (EUA) }\end{array}$ & 619 & $\begin{array}{l}\geq 49 \\
61,4 \pm 8,3\end{array}$ & $\begin{array}{l}\text { Sim/Não } \\
\text { (NA) }\end{array}$ & $\begin{array}{l}\text { DXA } \\
\text { (CL (L1-L4), CF e fêmur } \\
\text { total) }\end{array}$ \\
\hline $\begin{array}{l}\text { Schnatz et al. }{ }^{18} 2010 \\
\text { (EUA) }\end{array}$ & 619 & $\begin{array}{l}>49 \\
61,4 \pm 8,3\end{array}$ & $\begin{array}{l}\text { Um mês no mínimo de } \\
\text { lactação exclusiva } \\
\text { (NA) }\end{array}$ & $\begin{array}{l}\text { DXA } \\
\text { (CL (L1-L4), CF, fêmur } \\
\text { total) }\end{array}$ \\
\hline $\begin{array}{l}\text { Sioka et al.51 } 2010 \\
\text { (Europa) }\end{array}$ & 124 & $\begin{array}{l}40-79 \\
61,2 \pm 7,6\end{array}$ & $\begin{array}{l}\text { Maior ou menor de } 6 \mathrm{~m} \\
\text { (NA) }\end{array}$ & $\begin{array}{l}\text { DXA } \\
\text { (CL (L1- L4); quadril } \\
\text { esquerdo) }\end{array}$ \\
\hline
\end{tabular}

Reduz a DMO com aumento do tempo de lactação.

Observou aumento significativo na DMO relacionado à amamentação.

A lactação, mesmo prolongada, não possui efeito na DMO na pós-menopausa.

A DMO apresentou correlação negativa significativa com a duração da amamentação, após ajuste, sugerindo-a como fator de risco.

A chance de osteoporose foi significativamente menor entre mulheres que amamentaram.

Menor prevalência de osteoporose relacionada à amamentação.

Sem diferença significativa na DMO entre mulheres com DMO normal e as com osteoporose/ osteopenia com diferentes períodos de amamentação.

continua 
Características dos estudos observacionais que avaliaram o efeito da amamentação na massa óssea de mulheres na pós-menopausa.

\begin{tabular}{lllll}
\hline $\begin{array}{l}\text { Referência, ano } \\
\text { (Local do estudo) }\end{array}$ & N & $\begin{array}{l}\text { Idade (anos) } \\
\text { faixa etária e/ou } \\
\text { média (dp) }\end{array}$ & $\begin{array}{c}\text { História de lactação } \\
\text { (Tempo médio) }\end{array}$ & $\begin{array}{c}\text { Método (sítio de } \\
\text { mensuração) } \\
\text { Avaliação de precisão }\end{array}$ \\
\hline $\begin{array}{l}\text { Khoo et al.52 } 2011 \\
\text { (China) }\end{array}$ & 1654 & $\begin{array}{l}65-98 \\
72,7 \pm 5,4\end{array}$ & $\begin{array}{l}\text { Total em anos } \\
(3,1 \pm 3,4)\end{array}$ & DXA \\
(quadril total e CL (L1- L4))
\end{tabular}

\begin{tabular}{|c|c|c|c|c|}
\hline $\begin{array}{l}\text { Yilmaz et al.54 } 2012 \\
\text { (Turquia) }\end{array}$ & 1196 & $59,97 \pm 8,56$ & $\begin{array}{l}\text { Total em meses e } \\
\text { número de } \\
\text { mamadas/dia: } \\
(60,8 \pm 38,8 \text { - meses }) \\
(8,2 \pm 3,9 \text { - mamadas/dia) }\end{array}$ & DXA (CL e CF) \\
\hline $\begin{array}{l}\text { Okyay et al. } .552013 \\
\text { (Turquia) }\end{array}$ & 542 & $\begin{array}{l}\text { Sem } \\
\text { osteoporose: } \\
61,8 \pm 9,5 \\
\text { Com } \\
\text { osteoporose: } \\
60,6 \pm 10,2\end{array}$ & $\begin{array}{l}\text { Total em meses e } \\
\text { tempo/criança: } \\
(26,6 \pm 1,2 \text { - meses }) \\
(10,0 \pm 6,4 \text { - meses por } \\
\text { criança) }\end{array}$ & $\begin{array}{l}\text { DXA } \\
\text { (NA) }\end{array}$ \\
\hline
\end{tabular}

Tesvetov et al.29 $2014 \quad 294 \quad 30-55^{*}$

(Israel)
$47 \pm 6,4$
$9,12 \pm 11,1-$ meses*

(coluna, CF e quadril total)

Coeficiente de variação
Associação inversa significativa

entre o tempo de amamentação com a DMO. Porém, com poder preditivo fraco.

Maior probabilidade de osteopenia/osteoporose para mulheres que amamentaram entre 36 e 48 meses.

A amamentação não foi fator de risco independente para baixa DMO, embora tenha apresentado correlação inversa significativa com a DMO.

A duração da amamentação e o número de mamadas ao dia apresentaram correlação inversa entre a DMO na $\mathrm{CL}$ e no CF.

Mulheres com osteoporose que amamentaram apresentaram maiores médias de duração da amamentação total e por criança, em relação às mulheres sem osteoporose e que amamentaram.

A osteoporose também foi mais prevalente entre mulheres com idade gestacional menor, assim como foi mais frequente entre as que amamentaram por períodos maiores de um ano. Quando associados período por criança $\geq 1$ ano e idade gestacional < 27, comparados às demais condições, a chance de osteoporose foi maior.

A duração da amamentação se correlacionou de forma significante com menores valores da DMO, na pósmenopausa. Quanto maior a duração da amamentação menor a DMO em todos os sítios e mais intensa na coluna lombar.

continua 
Tabela 1

Características dos estudos observacionais que avaliaram o efeito da amamentação na massa óssea de mulheres na pós-menopausa.

\begin{tabular}{|c|c|c|c|c|c|}
\hline $\begin{array}{l}\text { Referência, ano } \\
\text { (Local do estudo) }\end{array}$ & $\mathbf{N}$ & $\begin{array}{l}\text { Idade (anos) } \\
\text { faixa etária e/ou } \\
\text { média (dp) }\end{array}$ & $\begin{array}{l}\text { História de lactação } \\
\text { (Tempo médio) }\end{array}$ & $\begin{array}{c}\text { Método (sítio de } \\
\text { mensuração) } \\
\text { Avaliação de precisão }\end{array}$ & Principais resultados \\
\hline $\begin{array}{l}\text { Yeo et al.30* } 2015 \\
\text { (Coreia) }\end{array}$ & 588 & $64 \pm 0,7$ & $\begin{array}{l}17,7 \pm 0,6 \text { - meses por } \\
\text { criança }\end{array}$ & $\begin{array}{l}\text { DXA } \\
\text { (CL - L1 e L4 e fêmur } \\
\text { proximal - quadril total) } \\
\text { Calibração equipamento }\end{array}$ & $\begin{array}{l}\text { A DMO do fêmur total, CF, e da } \\
C L \text { foi significativamente } \\
\text { menor em mulheres, na pós- } \\
\text { menopausa, com maior } \\
\text { duração da amamentação. }\end{array}$ \\
\hline Coorte & & & & & \\
\hline $\begin{array}{l}\text { Hansen et al.56 } 1991 \\
\text { (Dinamarca) }\end{array}$ & $\begin{array}{l}1997: 178 \\
1989: 121\end{array}$ & $\begin{array}{l}1977: 51 \pm 2 \\
1989: 63 \pm 2\end{array}$ & $\begin{array}{l}\text { Mínimo de duas } \\
\text { semanas } \\
(13 \pm 10 \text { meses })\end{array}$ & $\begin{array}{l}\text { SPA } \\
\text { (entre a ulna e o rádio) } \\
\text { DXA } \\
\text { (CL - L2 a L4 e CF, } \\
\text { trocanter e região de } \\
\text { Ward) } \\
\text { Calibração equipamento }\end{array}$ & $\begin{array}{l}\text { O CMO avaliado no braço } \\
\text { permaneceu significativamente } \\
\text { maior nas mulheres que } \\
\text { amamentaram em relação às } \\
\text { que não amamentaram ao } \\
\text { longo dos } 12 \text { meses. }\end{array}$ \\
\hline
\end{tabular}

* Inclui mulheres na pré e pós-menopausa

Adme $=$ anos desde a menopausa; $C F=$ colo do fêmur; $C L=$ coluna lombar; $C M O=$ conteúdo mineral ósseo; $\mathrm{DMO}=$ densidade mineral óssea; $\mathrm{dp}=$ desvio-padrão; DPA= absorciometria por dois fótons de raios: $\mathrm{DXA}=$ absorciometria por dupla emissão de raios $\mathrm{X}$; IC95\%: intervalo de confiança de $95 \%$; id= idade; IMC= índice de massa corporal; mdme= meses após a menopausa; NA= não apresentou; NS= não significativo; $\mathrm{PMO}=$ pico de massa óssea; $\mathrm{SPA}=$ absorciometria por um único fóton de raios; TC= tomografia computadorizada; $\mathrm{TRH}=$ terapia de reposição hormonal; OR= odds ratio.

Oito estudos 17,27,29-31,41,44,48 continham em sua população mulheres que não estavam na pósmenopausa, mas por apresentarem em seus resultados as análises em separado para nosso grupo-alvo, puderam ser incluídos.

Quanto a data de publicação, 14 $(41,2 \%) 26,27,31,33,39-47,56$ foram publicados até a década de 1990 e $20(58,8 \%)^{12,13,16-19,28-30,32,34,35,48-55}$ no ano $2000 \mathrm{em}$ diante. Considerando todos os estudos, 34.935 mulheres na pós-menopausa foram avaliadas. O tamanho da amostra variou de 63 a 9.704 mulheres, e $91,2 \%(n=31)$ dos estudos tinham mais de 100 participantes.

Quanto aos objetivos dos estudos, 21 artigos $(61,8 \%)^{12,13,16,17,26,29-32,34,40,42,44-46,53-55}$ tiveram como proposta central a avaliação da amamentação e sua relação tardia sobre a massa óssea em mulheres na pós-menopausa. Os demais estudos $(38,2 \%)^{18,19,27,28,33,35,39,41,43,47,51,52,56}$ avaliaram o efeito da amamentação na massa óssea como objetivo secundário, dentre outros fatores diversos.

\section{Mensuração da prática da amamentação}

Os estudos diferiram em suas metodologias para mensurar o histórico da amamentação. Parte deles considerou a variável "amamentação" de maneira dicotômica - sim/não $(20,6 \%) 16,28,33,35,39,41,43$ - e os demais, com base na soma do tempo total de prática da amamentação em meses, anos ou semanas $(79,4 \%)$. Ainda, entre estes últimos houve divergência na consideração do tempo total que durou a amamentação. Hansen et al.,56 por exemplo, avaliaram o tempo total de amamentação, excluindo casos cujo tempo era inferior a duas semanas, e também avaliaram o período por criança amamentada por no mínimo seis meses. Outro estudo avaliou a influência da duração de seis meses de lactação. ${ }^{51}$

A identificação do tipo de aleitamento foi escassa. Apenas dois trabalhos 16,46 avaliaram especificamente o tempo da amamentação exclusiva.

Outras formas de avaliação também foram contempladas, e não somente a duração. Dois trabalhos avaliaram a influência da duração da amamentação por cada filho.27,55 Yilmaz et al.54 avaliaram a influência do relato do número de mamadas ao dia e Osei-Hyiaman et al. ${ }^{47}$ avaliaram o relato de uso de fórmulas lácteas.

Parte dos estudos 27,47,54,55,56 agrupou diferentes informações sobre a prática da amamentação. Hu et al.,27 por exemplo, observaram maior densidade mineral óssea $(\mathrm{DMO})$ em relação à duração da 
amamentação, quando especificada por quantidade de crianças amamentadas e não somente quanto ao tempo total, em mulheres chinesas na pósmenopausa.

Instrumentos e sítios ósseos de avaliação da massa óssea

Quanto à mensuração do desfecho de interesse, ou seja, a massa óssea na pós-menopausa, todos os estudos a avaliaram por meio de densitometria óssea, utilizando diferentes equipamentos e diferentes áreas ósseas do corpo. Cinco (14,7\%) estudos usaram o SPA (absorciometria por um único fóton de raios);27,39,43,45,47 outros quatro $(11,8 \%)$ trabalhos utilizaram DPA (absorciometria por dois fótons de raios); $31,40,41,44$ dois $(5,9 \%)$ estudos mesclaram uso do SPA e DXA (absorciometria por dupla emissão de raios $\mathrm{X}) ; 42,56$ um $(2,9 \%)$ estudo fez uso da tomografia computadorizada 26 e os demais, do DXA $(n=22)(64,7 \%)$.

\section{Amamentação e massa óssea}

Entre os artigos analisados, $70,6 \%(\mathrm{n}=24)$ observaram que ter amamentado ou não está associado com a massa óssea na pós-menopausa: nove trabalhos $(26,5 \%)$ observaram aumento na DMO de mulheres que amamentaram, em relação àquelas de mesma idade que nunca amamentaram ou o fizeram por menos tempo. $16,18,27,28,39-41,44,56$ Os demais $(44 \% ; n=15)$ relataram redução na DMO associada à prática da amamentação ou sua maior duração. 12,17,19,26,29,30-32,47,48,50,52-55 Estudos se destacaram por informações mais específicas quanto à duração da amamentação, como Berning et al.,26 que observaram maior DMO em mulheres que amamentaram por mais de 24 semanas, em relação às que nunca amamentaram. Outro estudo 44 também mostrou relação direta entre DMO e amamentação de duração acima de oito meses. Em contrapartida, Kojima et al.48 relataram relação inversa entre amamentação e DMO em japonesas com faixa etária de 60 a 64 anos.

Em relação à doença óssea propriamente dita, Schnatz et al.,16,18 com base nos dados de dois estudos com mulheres norte-americanas, sugeriram que a amamentação poderia constituir um fator de proteção para a osteoporose na pós-menopausa. No entanto, Rojano-Mejía et al.,19 estudando mexicanas, observaram maior probabilidade de osteoporose e osteopenia entre mulheres na pósmenopausa cuja duração da amamentação foi entre 36 e 48 meses. Okyay et al.,55 em estudo na Turquia, registraram maior prevalência de osteoporose entre mulheres que relatavam amamentar por mais de um ano por criança.

\section{Susceptibilidade a vieses}

A Tabela 2, a seguir, descreve a susceptibilidade a vieses individual para cada estudo.

Nenhum estudo considerou integralmente o conjunto de variáveis potencialmente relevantes para o esclarecimento da relação da amamentação com a DMO na pós-menopausa: amamentação exclusiva, idade gestacional, tempo entre as gestações, ocorrência de gestação gemelar, hábito de fumo/álcool, anos desde a menopausa, prática de atividade física, uso de terapia de reposição hormonal, consumo de cálcio/suplementação, doenças que afetam metabolismo ósseo e medicamentos que afetam a saúde óssea. Foram frequentemente considerados: hábito de fumo/álcool, anos desde a menopausa e prática de atividade física.

Onze estudos atenderam, aproximadamente, a $50 \%$ dos itens de qualidade. $16,17,26,27,30,33,34,48,50,53,56$ com seis $17,26,30,48,50,53$ observações para associação inversa entre a amamentação e a DMO na pós-menopausa; dois não observaram associação 33,34 e os demais relataram associação direta. Sete artigos 18,19,31,45,49,52,54 atenderam a mais de $50 \%$ dos critérios de qualidade e, dentre estes, quatro referiram associação inversa; 19,31,52,54 outros dois 45,49 não encontraram associação e demais relataram associação direta da amamentação com a DMO na pós-menopausa.

\section{Discussão}

O propósito da presente revisão sistemática da literatura foi esclarecer o tipo de relação entre a prática da amamentação e a massa óssea de mulheres na pós-menopausa. Em nossa revisão, entretanto, constatamos a substancial heterogeneidade entre os estudos quanto à metodologia de mensuração da exposição e do desfecho, covariáveis consideradas, e especialmente, quanto aos seus objetivos centrais, dificultando a comparabilidade entre eles.

Quanto à amamentação, temos a importante recomendação da Organização Mundial da Saúde (OMS), 57 que incentiva o aleitamento materno de forma exclusiva até os seis meses e praticado pelo menos até os dois anos ou mais de idade da criança. No entanto, apenas dois trabalhos apreciaram a amamentação exclusiva. Um deles 46 não encontrou relação e o outro observou menor prevalência de osteoporose entre as mulheres que amamentaram. 16 
Tabela 2

Descrição da susceptibilidade a vieses dos estudos observacionais.

\begin{tabular}{|c|c|c|c|c|c|}
\hline \multirow[b]{2}{*}{ Referência, ano } & \multirow{2}{*}{$\begin{array}{c}\text { Critério de } \\
\text { elegibilidade } \\
\text { dos } \\
\text { participantes }\end{array}$} & \multirow[b]{2}{*}{ Etnia } & \multicolumn{2}{|c|}{ Amamentação } & \multirow{2}{*}{$\begin{array}{l}\text { Mensurou (idade gestacional -1; tempo entre as ges } \\
\text { tações -2; gestação gemelar -3; hábito fumo/álcool - } \\
\text { 4; anos desde a menopausa -5; prática de atividade } \\
\text { física -6; uso TRH -7; consumo de cálcio/suplemen- } \\
\text { tação -8; doenças que afetam o metabolismo ósseo } \\
\text { 9; medicamentos que afetam saúde óssea- 10) }\end{array}$} \\
\hline & & & Duração total & Exclusiva & \\
\hline Aloia et al.39 1983 & 1 & 1 & Não & Não & $\operatorname{Sim}(4 ; 5 ; 6 ; 7 ; 8 ; 9 ; 10)$ \\
\hline Hreshchyshyn et al.40 1988 & I & $\operatorname{Sim}$ & Não & Não & Não \\
\hline Stevenson et al.41 1989 & Não & $\operatorname{Sim}$ & Não & Não & $\operatorname{Sim}(4 ; 6 ; 8)$ \\
\hline Lissner et al.31 1991 & 1 & 1 & Sim & Não & $\operatorname{Sim}(1 ; 4 ; 5 ; 6 ; 7 ; 8 ; 9)$ \\
\hline Hansen et al.56 1991 & Sim & Não & $\operatorname{Sim}$ & Não & $\operatorname{Sim}(1 ; 4 ; 6 ; 8 ; 9 ; 10)$ \\
\hline Kritz-Silverstein et al.42 1992 & 1 & I & Sim & Não & $\operatorname{Sim}(4 ; 5 ; 10)$ \\
\hline Bauer et al.43 1993 & I & $\operatorname{Sim}$ & Não & Não & $\operatorname{Sim}(4 ; 5 ; 6 ; 7 ; 8 ; 9 ; 10)$ \\
\hline Berning et al.26 1993 & Sim & $\operatorname{Sim}$ & Sim & Não & $\operatorname{Sim}(4 ; 5 ; 8 ; 10)$ \\
\hline Melton III et al.44 1993 & Não & 1 & Sim & Não & $\operatorname{Sim}(5 ; 7 ; 8)$ \\
\hline Fox et al.45 1993 & Não & $\operatorname{Sim}$ & Sim & Não & $\operatorname{Sim}(1 ; 3 ; 4 ; 5 ; 6 ; 7 ; 8)$ \\
\hline Hu et al.27 1994 & Sim & Não & Sim & Não & $\operatorname{Sim}(4 ; 5 ; 6 ; 8)$ \\
\hline Orwoll et al.33 1996 & 1 & 1 & 1 & 1 & $\operatorname{Sim}(1 ; 4 ; 6 ; 7 ; 8 ; 10)$ \\
\hline Sinigaglia et al.46 1996 & I & $\operatorname{Sim}$ & Sim & 1 & $\operatorname{Sim}(4 ; 6 ; 7 ; 8 ; 9 ; 10)$ \\
\hline Osei-Hyiaman et al.47 1998 & Sim & $\operatorname{Sim}$ & Não & Sim & $\operatorname{Sim}(4 ; 5 ; 6 ; 8 ; 9 ; 10)$ \\
\hline Grainge et al.34 2001 & 1 & I & $\operatorname{Sim}$ & Não & $\operatorname{Sim}(4 ; 5 ; 7 ; 9 ; 10)$ \\
\hline Kojima et al.48 2002 & Sim & I & Sim & Não & $\operatorname{Sim}(5)$ \\
\hline Zhang et al.49 2003 & 1 & $\operatorname{Sim}$ & Sim & Não & $\operatorname{Sim}(1 ; 5)$ \\
\hline Gur et al.32 2003 & I & Não & Sim & Não & $\operatorname{Sim}(4 ; 5 ; 6 ; 7 ; 8 ; 9)$ \\
\hline Hassa et al.35 2005 & I & I & Não & Não & $\operatorname{Sim}(4 ; 5 ; 6 ; 7 ; 8 ; 10)$ \\
\hline Ozdemir et al.12 2005 & Não & Não & Sim & Não & $\operatorname{Sim}(1 ; 5)$ \\
\hline Dursun et al. 172006 & I & I & Sim & Não & $\operatorname{Sim}(4 ; 5 ; 6 ; 9 ; 10)$ \\
\hline Hill et al.28 2008 & 1 & $\operatorname{Sim}$ & Não & Não & $\operatorname{Sim}(4 ; 6 ; 9 ; 10)$ \\
\hline Lenora et al.13 2009 & I & Não & Sim & Não & $\operatorname{Sim}(4 ; 5 ; 10)$ \\
\hline Hosseinpanah et al.50 2010 & I & 1 & Sim & Não & $\operatorname{Sim}(4 ; 5 ; 7 ; 8 ; 9 ; 10)$ \\
\hline Schnatz et al.16 2010 & 1 & $\operatorname{Sim}$ & I & Não & $\operatorname{Sim}(4 ; 6 ; 7 ; 8 ; 9 ; 10)$ \\
\hline Schnatz et al.18 2010 & I & $\operatorname{Sim}$ & Não & $\operatorname{Sim}$ & $\operatorname{Sim}(1 ; 4 ; 6 ; 7)$ \\
\hline Sioka et al.51 2010 & $\mathrm{I}$ & Não & I & Não & $\operatorname{Sim}(5 ; 7 ; 9 ; 10)$ \\
\hline Khoo et al.52 2011 & I & $\operatorname{Sim}$ & Sim & Não & $\operatorname{Sim}(4 ; 6 ; 7 ; 8 ; 9 ; 10)$ \\
\hline Rojano-Mejia et al.19 2011 & Sim & $\operatorname{Sim}$ & Sim & Não & $\operatorname{Sim}(4 ; 5 ; 6 ; 7 ; 8 ; 9 ; 10)$ \\
\hline Yazici et al.53 2011 & I & 1 & Sim & Não & $\operatorname{Sim}(5 ; 6 ; 7 ; 9 ; 10)$ \\
\hline Yilmaz et al.54 2012 & Sim & I & Sim & Não & $\operatorname{Sim}(4 ; 9 ; 10)$ \\
\hline Okyay et al.55 2013 & Sim & 1 & Sim & Não & $\operatorname{Sim}(1 ; 4 ; 5 ; 9 ; 10)$ \\
\hline Tsvetov et al.29 2014 & Sim & Não & Sim & Não & $\operatorname{Sim}(5 ; 6 ; 8)$ \\
\hline Yeo et al.30 2015 & Sim & Não & Sim & Não & $\operatorname{Sim}(4 ; 5 ; 6 ; 8)$ \\
\hline
\end{tabular}

I = incompleto; TRH= terapia de reposição hormonal. 
Alguns estudos destacaram determinados períodos de amamentação e sua relação com a DMO na pós-menopausa. Berning et al. 26 observaram aumento da DMO somente entre as mulheres com relato de amamentação acima de 24 semanas (seis meses). Já Sioka et al. 51 não encontraram diferença na DMO de mulheres com osteoporose ou osteopenia em relação às mulheres com DMO normal com o mesmo tempo de amamentação (maior ou menor que seis meses). Outro trabalho relatou que a amamentação acima de oito meses demonstrou associação significativa com a maior DMO.44 Contudo, nenhum dos estudos acima relatou se houve amamentação exclusiva ou complementada. Por outro lado, Rojano et al. ${ }^{19}$ observaram maior chance de osteoporose e osteopenia entre mulheres com relato de amamentação entre 36 e 48 meses. Kojima et al. 48 observaram relação inversa entre o tempo de amamentação e a DMO de mulheres na faixa etária de 60-64 anos. Gur et al.32 compararam a DMO de mulheres que não amamentaram com as que o fizeram por oito meses ou mais e observaram que a DMO decresceu conforme o tempo de amamentação aumentou.

Ainda merece atenção outro fato pouco estudado: a duração do tempo de amamentação por filho. $27,30,55 \mathrm{Hu}$ et al.27 referem correlação positiva entre a exposição e o desfecho, quando considerado o tempo total de amamentação por criança; diferentemente, Okyay et al.55 relataram maior prevalência de osteoporose entre as mulheres que amamentaram mais de um ano por filho. Esta informação, portanto, precisa ser considerada em futuros trabalhos.

Outro fato referente à amamentação é a influência exercida pela intensidade e duração da lactação sobre a mobilização óssea materna. O volume de leite produzido exerce maior impacto sobre a DMO materna do que a quantidade de cálcio perdida ao longo dos seis primeiros meses de amamentação. ${ }^{58}$ Apenas um trabalho investigou essa intensidade (número de mamadas/dia) e observou que tanto a duração quanto o número de mamadas se relacionaram com a redução da DMO na pósmenopausa. 54

Apenas no estudo de Osei-Hyiaman et al.47 foram comparadas a DMO na pós-menopausa de mulheres que somente amamentaram, com as que só utilizaram fórmula láctea para seus filhos e as que ofereceram ambos. Os autores observaram que a DMO foi maior no grupo que relatou uso de ambos, seguido das que só ofereceram leite materno.

Em relação aos sítios ósseos de maior mobilização durante a amamentação, tem-se que a maior perda de DMO ocorre na parte trabecular das estruturas ósseas. Berning et al., 26 por meio da tomografia computadorizada, referiram que a DMO na área trabecular da coluna lombar de mulheres na pós-menopausa associou-se positivamente com a duração da amamentação, confirmando-o como sítio ósseo de mobilização durante a lactação e que, assim, deve ser considerado em pesquisas futuras.

De acordo com a literatura, a DMO perdida na amamentação é recuperada aos níveis pré-gestacionais alguns meses após o desmame em estudos com mulheres adultas saudáveis. Desta forma, condições específicas merecem ser avaliadas, pois podem justificar a associação entre a fase da amamentação com a massa óssea tardia. São elas: gestação na fase da adolescência, carência de vitamina D, múltiplas gestações e seus intervalos, gestação gemelar e gestação em torno dos 40 anos de idade. Acredita-se que estas sejam situações em que a adaptação normal à gestação esteja comprometida, inclusive a recuperação da massa óssea perdida durante a amamentação.59 Entretanto, apenas seis estudos avaliaram a idade materna na primeira gestação (não adolescentes). Dentre eles, metade afirma relação significativa entre a idade materna na primeira gestação e a DMO na pósmenopausa, $14,18,55$ sendo um relato negativo quando a idade da primeira gestação foi precoce;55 os demais divergiram quando a idade materna da primeira gestação foi mais tardia.12,16 Somente cinco estudos consideram a vitamina $\mathrm{D}$ dentre as variáveis de risco18,33,43,55,56 e nenhum estudo avaliou gestação gemelar ou espaço entre as gestações. Há outros fatores (hábito de fumo e consumo de álcool e de cafeína, idade da menarca, duração da menopausa, prática de atividade física, uso de terapia de reposição hormonal, idade, consumo de cálcio ou suplementação, uso de medicamentos ou ocorrência de doenças que afetam a saúde óssea e menopausa por cirurgias ginecológicas) explicativos da redução da DMO na pós-menopausa que potencialmente poderiam interferir na relação estudada. 14,59,60 Nenhum estudo considerou todos esses diversos fatores em suas análises. Em relação à etnia, observou-se o cuidado em identificá-la em 20 estudos $(58,8 \%)$. Dos sete estudos que identificaram sua amostra como constituída por mulheres brancas, 16,18,19,33,39,41,43 três relataram associação direta. 16,18,39 Hill et al.,28 autores do único estudo entre mulheres negras, observaram maior DMO entre as que relataram ter amamentado. Rojan-Mejía et al.19 estudaram "mestiças" e observaram maior probabilidade de osteoporose e osteopenia entre as mulheres que amamentaram de 36 a 48 meses na 
vida - outro fator a ser mais bem estudado.

Dentre os trabalhos que relataram associação entre a amamentação e a massa óssea na pósmenopausa $(\mathrm{n}=24)$, mais da metade $(64 \%, \mathrm{n}=16)$ tinham como objetivo central avaliar de forma mais específica a amamentação, por isso são mais esclarecedores quanto à investigação de interesse. Dentre eles, $62,5 \%(n=15)$ observaram redução da massa óssea na pós-menopausa, e os demais o inverso $(n=9)$. Ainda assim, são limitados na mensuração da amamentação e na consideração de fatores que foram elencados na avaliação da qualidade como potencialmente confundidores da relação.

O único estudo de coorte identificado não teve o

\section{Referências}

1. Lima-Costa MF, Veras R. Saúde pública e envelhecimento. Cad Saúde Pública. 2003; 19: 700-1.

2. WHO Study Group on Assessment of Fracture Risk and its Application to Screening for Postmenopausal Osteoporosis. Assessment of fracture risk and its application to screening for postmenopausal osteoporosis: report of a World Health Organization Study Group. World Health Organ Tech Rep Ser. 1994; 843: 129.

3. Black DM, Arden NK, Palermo L, Pearson J, Cummings SR. Prevalent vertebral deformities predict hip fractures and new vertebral deformities but not wrist fractures. Study of osteoporatic fractures research group. J Bone Miner Res. 1999; 14: 821-8.

4. Araujo DV, Oliveira JH, Bracco OL. Custo da fratura osteoporótica de fêmur no Sistema Suplementar de Saúde Brasileiro. Arq Bras Endocrinol Metabol. 2005; 49: 897.

5. Burge R, Dawson-Hughes B, Solomon DH, Wong JB, King A, Tosteson A. Incidence and economic burden of osteoporosis-related fractures in the United States, 2005-2025. J Bone Miner Res. 2007; 22: 465-75.

6. Siris ES, Chen YT, Abbott TA, Barrett-Connor E, Miller PD, Wehren LE, Berger ML. Bone mineral density thresholds for pharmacological intervention to prevent fractures. Arch Intern Med. 2004; 164: 1108-12.

7. Sornay-Rendu E, Munoz F, Garnero P, Duboeuf F, Delmas PD. Identification of osteopenic women at high risk of fracture: the OFELY study. J Bone Miner Res. 2005; 20:1813-

8. Cole R. Postmenopausal osteoporosis: Another approach to management. J Fam Pract. 2010; 59: E1-E7.

9. Rockville MD. Bone health and osteoporosis: a report of the Surgeon General. United States: Department of Health and Human Services; 2004

10. Frazão P, Naveira M. Prevalência de osteoporose: uma revisão crítica. Rev Bras Epidemiol. 2006; 9: 206-14

11. Franucucci CM, Romagni P, Camilletti A, Fiscaletti P,Amoroso L, G, Morbidell Ci, Boscaro M. Effect of natura early menopause on bone mineral density. Maturitas. 2008; 59: $323-8$ objetivo central de avaliar a amamentação sobre a DMO na pós-menopausa, por isso variáveis importantes em meio a esta relação não foram consideradas (idade da primeira gestação, espaço entre as gestações e gestações gemelares). A própria aferição do histórico da amamentação não foi detalhada.

Conclui-se que a associação entre a prática de amamentação e a massa óssea tardia ainda carece de elucidação, tendo em vista as limitações dos estudos já realizados, relacionadas principalmente a qualidade da mensuração da exposição e do desfecho, e à avaliação e controle de outros fatores de risco. Estudos futuros deverão ser planejados de modo a superar essas limitações.

12. Ozdemir F, Demirbag D, Rodoplu M. Reproductive factors affecting the bone mineral density in postmenopausal women. Tohoku J Med. 2005; 205: 277-85.

13. Lenora J, Lekamwasam S, Karlsson MK. Effects of multiparity and prolonged breastfeeding on maternal bone mineral density: a community-based cross-sectional study. BMC Women's Health. 2009; 9: 19.

14. Bezerra FF, Mendonça LM, Lobato EC, O'Brien KO, Donangelo CM. Bone mass is recovered from lactation to postweaning in adolescent mothers with low calcium intakes. Am J Clin Nutr. 2004; 80: 1322-6.

15. Chantry CJ, Auinger P, Byrd RS. Lactation among adolescent mothers and subsequent bone mineral density. Arch Pediatr Adolesc Med. 2004; 158: 650-6.

16. Schnatz PF, Barker KG, Marakovits KA, O'Sullivan DM. Effects of age at first pregnancy and breast-feeding on the development of postmenopausal osteoporosis. Menopause. 2010; 17: 1161-6.

17. Dursun N, Akin S, Dursun E, Sade I, Korkusuz F. Influence of duration of total breast-feeding on bone mineral density in a Turkish population: does the priority of risk factors differ from society to society? Osteoporos Int. 2006; 17: $651-5$.

18. Schnatz PF, Marakovits KA, O'Sullivan DM. Assement of postmenopausal women and significant risk factors for osteoporosis. Obstet Gynecol Surv.2010; 65: 591-6.

19. Rojano-Mejía D, Aguilar-Madrid G, López-Medina G, Cortes-Espinosa L, Hernández-Chiu MC, Canto-Cetina T, Vergara-López A, Coral-Vázquez RM, Canto P. Risk factors and impact on bone mineral density in postmenopausal Mexican mestizo women. Menopause. 2011; 18: 302-6.

20. Vieira JGH. Considerações sobre os marcadores bioquímicos do metabolismo

ósseo e sua utilidade prática. Arq Bras Endocrinol Metab.1999; 43: 415-22.

21. Kalkwarf HJ. Hormonal and dietary regulation of changes in bone density during lactation and after weaning in women. J Mammary Gland Biol Neoplasia. 1999; 4: 31929 
22. Prentice A, Jarjou LM, Stirling DM, Buffenstein R, Fairweather-Tait S. Biochemical markers of calcium and bone metabolism during 18 months of lactation in Gambian women accustomed to a low calcium intake and in those consuming a calcium supplement. J Clin Endocrinol Metab. 1998; 83: 1059-66

23. Kalkwarf HJ. Lactation and maternal bone health. Adv Exp Med Biol. 2004; 554: 101-14.

24. Ritchie LD, Fung EB, Halloran BP, Turnlund JR, Van Loan MD, Cann CE, King JC. A longitudinal study of calcium homeostasis during human pregnancy and lactation and after resumption menses. Am J Clin Nutr. 1998; 67: 693701.

25. Watts NB, Lewiecki EM, Miller PD, Baim S. National Osteoporosis Foundation 2008 Clinician`s Guide to Prevention and Treatment of Osteoporosis and the World Health Organization Fracture Risk Assement Tool (FRAX) what they mean to the bone densitometrist and bone technologist. J. Clin. Densitom. 2008; 11 (4): 473-7.

26. Berning B, van Kuijk C, Schütte HE, Kuiper JW, Drogendijk AC, Fauser BC. Determinants of lumbal bone mineral density in normal weight, non-smoking women soon after menopause. A study using clinical data and quantitative computed tomography. Bone and Mineral. 1993; 21: 129-39.

27. Hu JF, Zhao XH, Chen JS, Fitzpatrick J, Parpia B, Campbell TC. Bone density and lifestyle characteristics in premenopausal and postmenopausal Chinese women. Osteoporosis Int. 1994; 4: 288-97.

28. Hill DD, Cauley JA, Bunker CH, Baker CE, Patrick AL, Beckles GL, Wheeler VW, Zmuda JM. Correlates of bone mineral density among postmenopausal women of african Caribbean ancestry: Tabago women's health study. Bone. 2008; 43: 156-61.

29. Tsvetov G, Levy S, Benbassat C, Shraga-Slutzky I, Hirsch D. Influence of number of deliveries and total breastfeeding time on bone mineral density in premenopausal and Young postmenopausal women. Maturitas. 2014; 77: 24954

30. Yeo UH, Choi CJ, Choi, WS, Kim KS. Relationship between breastfeeding and bone mineral density among Korean women in the 2010 Korea National Health and Nutrition Examination Survey. J Bone Miner Metab. 2015; 2

31. Lissner L, Bengtsson C, Hansson T. Bone mineral content in relation to lactation history in pre- and postmenopausal women. Calcif Tissue Int. 1991; 48: 319-25.

32. Gur A, Cevik R, Nas K, Sarac AJ, Ataoglu S, Karakoc M, Can A, Gurkan F. The influence of duration of breastfeeding on bone mass in postmenopausal women of different age groups. Int J Clin Pract. 2003; 57: 82-6.

33. Orwoll ES, Bauer DC, Vogt TM, Fox KM. Axial bone mass in older women. Ann Intern Med. 1996; 124: 187-96

34. Grainge MJ, Coupland CA, Cliffe SJ, Chilvers CE, Hosking DJ. Reproductive, menstrual and menopausal factors: which are associated with bone mineral density in early postmenopausal women? Osteoporos Int. 2001; 12: 777-87.

35. Hassa H, Tanir HM, Senses T, Oge T, Sahin-Mutlu F. Related factors in bone mineral density of lumbal and femur in natural postmenopausal women. Arch Gynecol
Obstet. 2005; 273: 86-9.

36. Salari, Pooneh, Mohammad Abdollahi. The influence of pregnancy and lactation on maternal bone health: a systematic review. J Family Reprod Health. 2014; 8: 135-48.

37. Liberati A, Altman DG, Tetzlaff J, Mulrow C, Gotzsche PC, Loannidis JPA, Clarke M, Devereaux PJ, Kleijnen J, Moher D. The PRISMA statement for reporting systematic reviews and meta-analyses of studies that evaluate healthcare interventions: explanation and elaboration. Brit Med J. 2009; 339: b2700.

38. Malta M, Cardoso LO, Bastos FI, Magnanini MMF, Silva CMFP. Iniciativa STROBE: subsídios para a comunicação de estudos observacionais. Rev Saúde Pública. 2010; 44: 559-65.

39. Aloia JF, Vaswani AN, Yeh JK, Ross P, Ellis K, Cohn SH Determinants of bone mass in postmenopausal women. Arch Intern Med. 1983; 143: 1700-4

40. Hreshchyshyn MM, Hopkins A, Zylstra S, Anbar M. Associations of parity, breast-feeding, and birth control pills with lumbar spine and femoral neck bone densities. Am J Obstet Gynecol. 1988; 159: 318-22.

41. Stevenson JC, Lees B, Devenport M, Cust MP, Ganger KF. Determinants of bone density in normal women: risk factors for future osteoporosis? Brit Med J. 1989; 298: 924-

42. Kritz-Silverstein D, Barret-Connor E, Hollenbach KA. Pregnancy and lactation as determinants of bone mineral density in postmenopausal women. Am J Epidemiol. 1992; 136: 1052-9

43. Bauer DC, Browner WS, Cauley JA, Orwoll ES, Scott JC, Black DM, Tao JL, Cummings SR. Factors associated with appendicular bone mass in older women. Ann Intern Med.1993; 118: 657-65.

44. Melton III LJ, Bryant SC, Wahner HW, O`Fallon WM, Malkasian GD, Judd HL, Riggs BL. Influence of breastfeeding and other reproductive factors on bone mass later in life. Osteoporosis Int. 1993; 3: 76-83.

45. Fox KM, Magaziner J, Sherwin R, Scott JC, Plato CC, Nevitt M, Cummongs S. Reproductive correlates of bone mass en elderly women, Study of osteoporotic fractures research group. J Bone Res. 1993; 8: 901-8.

46. Sinigaglia L, Varenna M, Binelli L, Gallazzi M, Calori G, Ranza R. Effect of lactation on postmenopausal bone mineral density of the lumbar spine. J Reprod Med. 1996; 41: 439-43

47. Osei-Hyiaman D, Satoshi T, Ueji M, Hideto T, Kano K. Timing of menopause, reproductive years, and bone mineral density. A cross-sectional study of postmenopausal Japanese women. Am J Epidemiol. 1998; 148: 1055-61.

48. Kojima N, Douchi T, Kosha S, Nagata Y. Cross-sectional study of the effects of parturition and lactation on bone mineral density later in life. Maturitas. 2002; 41: 203-9.

49. Zhang YY, Liu PY, Deng HW. The impact of reproductive and menstrual history on bone mineral density in Chinese women. J Clin Densitom. 2003; 6: 289-96.

50. Hosseinpanah F, Sorouri M, Rambod M, Azizi F. Total duration of breastfeeding is associated with low bone mineral density in Iranian postmenopausal women. Int $\mathbf{J}$ Endocrinol Metab. 2010; 8: 153-8 
51. Sioka C, Fotopoulos A, Georgiou A, Xourgia X, Papadopoulos A, Kalef-Ezra JA. Age at menarche, age at menopause and duration of fertility as risk factors for osteoporosis. Climateric. 2010; 13: 63-71.

52. Khoo CC, Woo J, Leung PC, Kwok A, Kwok T. Determinats of bone mineral density in older postmenopausal chinese women. Climateric. 2011; 14: 378-83.

53. Yazici S, Korkmaz U, Erkan M, Korkmaz N, Baki AE, Alçelik A, Önder E, Ataoğlu S. The effect of breast-feeding duration on bone mineral density in postmenopausa Turkish women: a population-based study. Arch Med Sci. 2011; 7: 486-92.

54. Yılmaz H, Erkin G, Polat HAD, Küçükşen S, Sallı A,Uğurlu H. Reproductive Factors on bone mineral densitometry. Turk J Osteoporos. 2012; 18: 8-12.

55. Okyay DO, Okyay E, Dogan Em Kurtulmus S, Acet F, Taner CE. Prolonged breast-feeding is an independent risk factor for postmenopausal osteoporosis. Maturitas. 2013; 74: $270-5$

Recebido em 17 de março de 2015

Versão final apresentada em 01 de junho de 2015

Aprovado em 01 de julho de 2015
56. Hansen MA, Overgaard K, Riis BJ, Christiasen C. Potential risk factors for development of postmenopausal osteoporosis - Examined over a 12-year period. Osteoporosis Int. 1991; 1: 95-102.

57. OPAS/OMS (Organização Pan Americana da Saúde/Organização Mundial da Saúde). Amamentação. 2003 [acesso em 29 ago 2012]. Disponível em: $<$ http://www.opas.org.br/sistema/fotos/ amamentar.pdf $>$

58. Laskey MA, Prentice A, Hanratty LA, Jarjou LMA, Dibba B, Beavan SR, Cole TJ. Bone changes after 3 month of lactation: Influence of calcium intake, breast milk output and vitamin D-receptor genotype. Am J Clin Nutr. 1998; 67: 685-92.

59. Ward KA, Adams JE, Mughal M Z. Bone status during adolescence, pregnancy and lactation. Curr Opin Obstet Gynecol. 2005; 17 (4): 435-9.

60. Anesto JB, Cagigas A, Jorna AR. Influencia Del estilo de vida y los hábitos nutricionaçes sobre la calidad de masa ósea em mujeres postmenopáusicas. Ev Esp Comunit. 2005; 11: $80-8$. 